\title{
Analysis of Rotationally Symmetrical Antenna with Wider Beamwidth
}

\author{
Daisuke YAGYU×. Kazumasa TANAKA, Takafumi FU.JIMOTO \\ and Mitsuo TAGUCHI \\ Faculty of Engineering, Nagasaki University \\ 1-14 Bunkyo-machi, Nagasaki-shi, 852-8131, Japan
}

\section{Introduction}

The microstrip antcmua(MSA) is widcly used for small-sized tcrminal of mobilc communication system, as it is thin and light-weighted and it has larger gain as compared with wire antcmias. In some applications such as Global positioning system or Iridium satellite communication system which use a group of low-altitude, orbiting satellites, the wider benmwidth is desired for the antenna mounted on a terminal. In order to widen bearnwidth of MSA, the conformal MSA on a cylindrical conductor[1] or a spherical body[2] were proposed and analyzed. The anthors has proposed an oblate spheroidal antemna(OSA) in order to widen its beamwidth and has shown the analytical method[3]. In this paper, this antemna is muncrically and experimentally analy\%ed. OSA consists a lincarly polarized circular patch MSA and upper and lower oblate spheroidal conductors. The substratc of MSA is truncated at the edge of patch. In the numerical analysis, the electromagnetic fields within the antennic cavity are expanded by the modal functions and their unknown expansion coefficients are determined by the electric and magnetic field integral equations on the antenna surface.

\section{Formulation}

Fig. 1 shows the analytical model and its coordinate system. The cylindrical coordinate system $(\rho, \phi, z)$ is used within the MSA, and the oblate spheroidal coordinate system $(u, v, \phi)$ on the outer surface of conductor. The radius of patch is $a$ and the height of conductor is $c$. The cross section of oblate spheroidal conductor is the same as the patch. The antenna is excited betwecn the upper and lower conductors at the feed point $\rho=b, \phi=0$. The thickness and the dielectric constant of dielectric substratc are $D$ and $\epsilon_{1}=\epsilon_{r} \epsilon_{0}$, respectively. $\epsilon_{0}$ and $\mu_{0}$ are the diclectric constant and the permeability in free space, respectively. $S_{a}$ and $S_{c}$ : denote the aperture of substrate and the conductor surface, respectively.

The thickness of substrate is assumed to be much smaller than the wavelength. Then the electromagnetic fields within the substrate do not vary with the perpendicular direction to the patch, and can be obtained by the cavity model. By applying the ecfuivalence principle to this antema, the auxiliary problems in the internal and external regions of antemua are obtained[4]. Within the substrate, the clcctromagnctic ficlds arc expanded by the modal functions. The cquivalcnt elcetric and magnetic currents $J_{a}$ and $M$ on $S_{a}$ are equal to the tangential component of magnetic and electric fields within the substrate, respectively;

$$
J_{a}(a, \phi)=-\frac{j k_{1}}{\omega \mu_{0}} \sum_{n=0}^{N}\left\{B_{n} J_{n}{ }^{\prime}\left(k_{1} a\right)+\frac{j \omega \mu_{0} I_{0}}{2\left(1+\delta_{n}\right)} J_{n}\left(k_{1} b\right) N_{n}{ }^{\prime}\left(k_{1} a\right)\right\} \cos (n \phi) \boldsymbol{i}_{z}
$$




$$
M(a, \phi)=\sum_{n=0}^{N}\left\{B_{n} J_{n}\left(k_{i} a\right)+\frac{j \omega \mu_{0} I_{0}}{2\left(1+\delta_{n}\right)} J_{n}\left(k_{i} b\right) N_{n}\left(k_{i 1} a\right)\right\} \cos (n, \phi) \boldsymbol{i}_{\dot{q}}
$$

$J_{n}$ and $N_{n}$ are Bessel and Neumann functions of order $n$ and the prime denotes the derivative with respect to the argunent. $I_{0}$ is the total current at the feed point. $\delta_{n}$ is defined as $\delta_{n}=1$ for $n=0$ and $\delta_{n}=0$ for $n \neq 0$.

The electric courrent $J_{c:}$ on $S_{c}$ is assumed as follows:

$$
\boldsymbol{J}_{v}(u, v, \phi)=\left\{\begin{array}{rr}
\sum_{m=0}^{M} \sum_{n=0}^{N} A_{m r_{n}} \cos (m v) \cos (n \phi) \boldsymbol{i}_{v}+\sum_{m=0}^{M} \sum_{n=1}^{N} C_{m n} \cos (m v) \sin (n \phi) \boldsymbol{i}_{\dot{\phi}} \\
\sum_{m=0}^{M} \sum_{n=0}^{N} A_{m n} \cos (m v) \cos (n \phi) \boldsymbol{i}_{v}-\sum_{m=0}^{M} \sum_{n=1}^{N} C_{m n} \cos (m v) \sin (n \phi) \boldsymbol{i}_{\dot{\phi}} \\
; v \leq 0
\end{array}\right.
$$

wherc, $\left\{B_{n}\right\},\left\{A_{m n}\right\},\left\{C_{m n}\right\}$ are unknown cocfficients to be detcrmined from the integral ecuations on the antenna surface.

In the external region of antenna, the electromagnetic fields are cxpressed in tcrms of the equivalcnt clectric and magnetic currents $J_{a}$ and $M$ on $S_{a}$ and the electric current $J_{c}$ on $S_{c}$.

The magnetic field integral equation is formulated from the continuity condition on the tangential component of magnetic field on $S_{0}$;

$$
\begin{aligned}
\boldsymbol{i}_{z} \cdot[ & \frac{1}{2 \pi} n \times f_{S_{a}}\left\{-j \omega \epsilon_{0} M \psi+J_{a} \times \nabla^{\prime} \psi-\frac{1}{j \omega \mu_{0}}\left(\nabla^{(2)^{\prime}} \cdot M\right) \nabla^{\prime} \psi\right\} d S^{\prime} \\
& \left.+\frac{1}{2 \pi} n \times \int_{S_{c}}\left(J_{c} \times \nabla^{\prime} \psi\right) d S^{\prime}\right] \\
= & -\frac{j k_{i}}{\omega \mu_{0}} \sum_{n=0}^{N}\left\{B_{n} J_{n}{ }^{\prime}(k, \rho)+\frac{j \omega \mu_{0} I_{0}}{2\left(1+\delta_{n}\right)} J_{n}(k, b) N_{n}{ }^{\prime}\left(k_{i} \rho\right)\right\} \cos (n \phi)
\end{aligned}
$$

where, $\nabla^{(2)^{\prime}}$. denotes the surface divergence. $n$ is the unit vector normal to the antenna surface. $\psi$ is Green's function. The electric field integral equation is derived from the boundary condition that the tangential component of electric ficld vanishes on $S_{c}$;

$$
\begin{aligned}
\frac{1}{2 \pi} n \times & {\left[\int_{S_{a}^{\prime}}\left\{-j \omega \mu_{0} J_{a} \psi-M \times \nabla^{\prime} \psi-\frac{1}{j \omega \epsilon_{0}}\left(\nabla^{(2))^{\prime}} \cdot J_{a}\right) \nabla^{\prime} \psi\right\} d S^{\prime}\right.} \\
+ & \left.f_{S_{c}}\left\{-j \omega \mu_{0} J_{c} \psi-\frac{1}{j \omega \epsilon_{0}}\left(\nabla^{(2)^{\prime}} \cdot J_{c}\right) \nabla^{\prime} \psi\right\} d S^{\prime}\right]=0
\end{aligned}
$$

The equivalent electric and magnetic currents on $S_{a}$ and the electric current on $S_{c}$ arc calculated by applying Galcrkin's incthod to these integral cejuations. The input, impedance is defined as a half of the voltage at feed point divided by the total current according to the clefinition of input impedance in the measurement.

\section{Results and Discussion}

Fig. 2 shows the calculated and measured input impcdances of OSA. The radius of patch $a$ is $15 \mathrm{~mm}$, and the feed point is located at $b=10.0 \mathrm{~mm}$. The thickness $D$ and 
the relative dielectrice constant $\epsilon_{r}$ of substrate is $1.528 \mathrm{~mm}$ and 2.15 , respectively. In the numcrical calculation, the ratio of height to radius of oblate spheroidal conductor is chosen as $c / a=0.05$ and 0.95 . The number of expansion mode is chosen as $M=N=3$. In the measurement, the circular patch MSA with the substrate truncated at the edge of patch is used. This antenna is mounted on the ground plane of $14 \mathrm{~cm} \times 14 \mathrm{~cm}$ and coaxially fed from this plane. Relatively good agreement is observed for between the calculated $(c / a=0.05)$ and the measured $(c / a=0)$ results.

Fig. 3 shows the calculatcd radiation pattcrns of OSA in E- and H-plancs. In E-plane, the beamwidth becomes wider as the height of oblate spheroidal conductor becomes higher. The vertical componcnt of current on $S_{0}$ becomes larger as the height, of oblate spheroidal conductor becomes higher. Therefore, the intensity of radiation field at the low angle of elevation in E-plane becomes larger. In H-plane, however, little difference is found on radiation patterns with $c / a=0.05$ and 0.05 .

\section{Conclusion}

The circular pateh MSA inserted in the center of oblate spheroidal conductor has been numerically and experimentally analyzed. The electromagnetic fields within the MSA are expressed as the summation of modal functions in the cylindrical coordinatc systcm. Their unknown cocfficicnts and the equivalcnt clectric and magnetic currents on antenna surface are determined by the magnetic field integral equation on the apcrture of substrate and the clectric ficld intcgral equation on the conductor surface. This antenna has wider beanwidth in E-plane as comparcd with circular patch MSA. Also, the bandwidth becomes wider and the resonant frequency becomes lower as the height of oblate spheroidal conductor becomes higher.

\section{References}

[1] K.-M.Luk ct al.:"Anlalysis of the cylindrical-rectangular patch antenna". IEEE Trans., AP-37. 2, pp.143-147, Feb. 1989

[2] K.-M.Luk et al.: "Patch antennas on splicrical body", Proc. IEE, 138, 1, pp.103-108, Feb. 1991

[3] D.Yagyu et al.:"Analysis of microstrip antenna with wider beamwidth", Proc. ICARSM, Voronczh, Russia, vol. 1, pp.6269, May 1997

[4] C.A.Balanis:"Antenna theory: Analysis and design", pp.447-454, John Wiley \& Sons, 1982

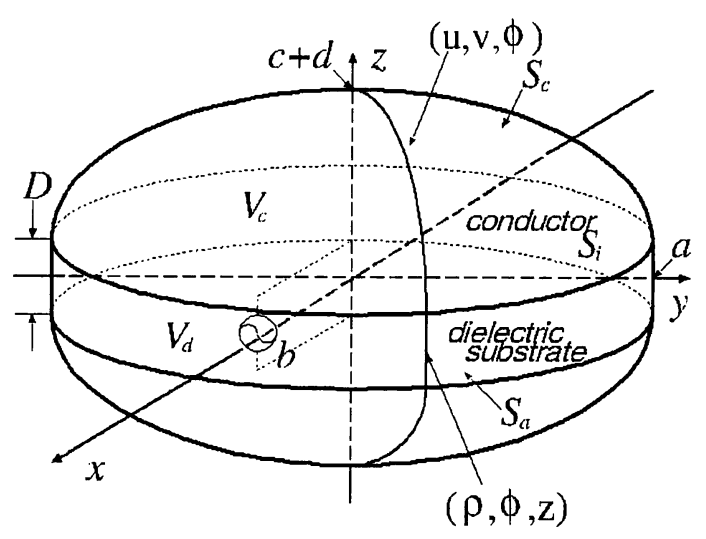

Fig. 1: Analytical model 


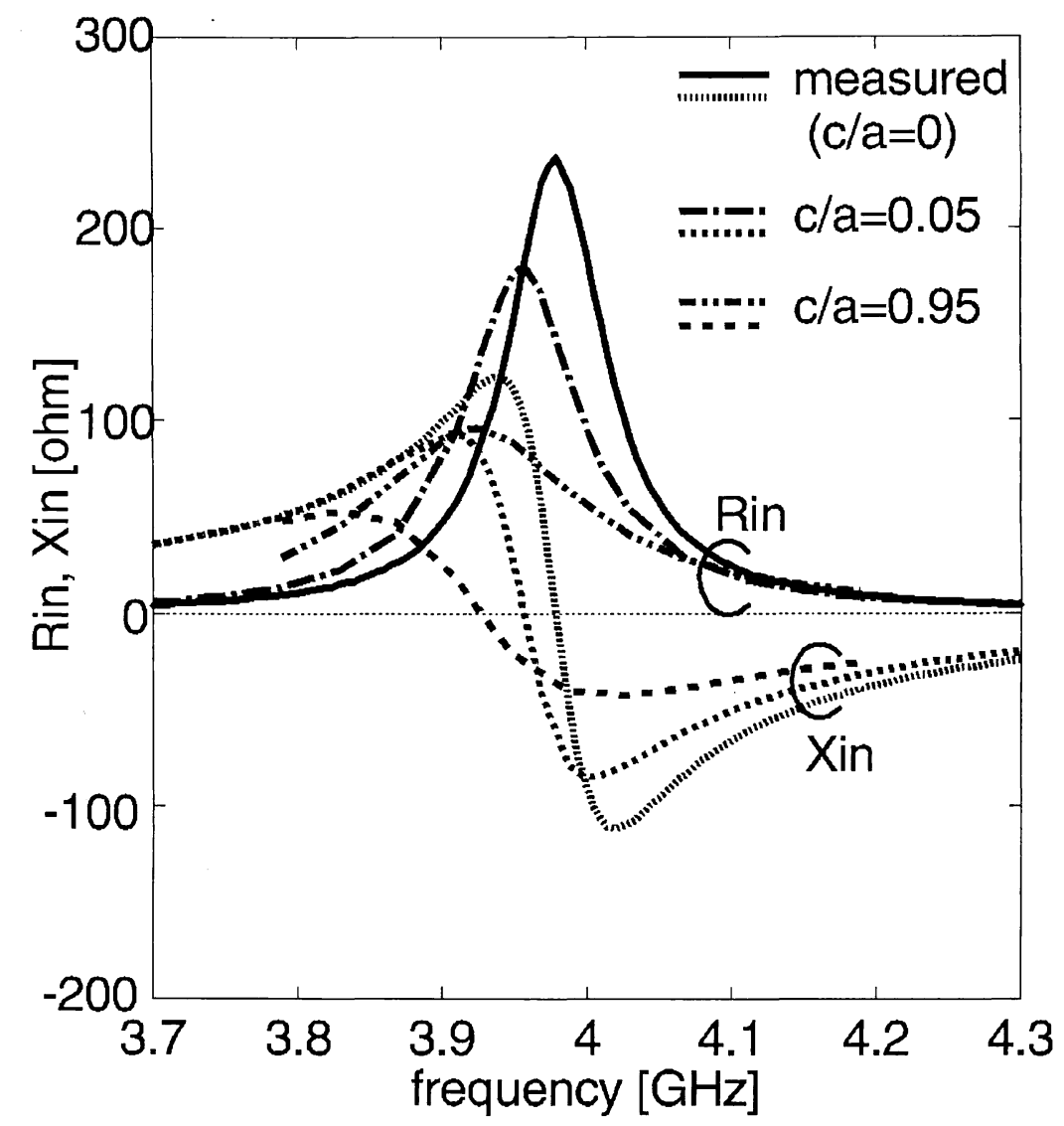

Fig. 2: Input Impedances

$a=15 \mathrm{~mm}, b=10.0 \mathrm{~mm}, D=1.528 \mathrm{~mm}, \epsilon_{r}=2.15$

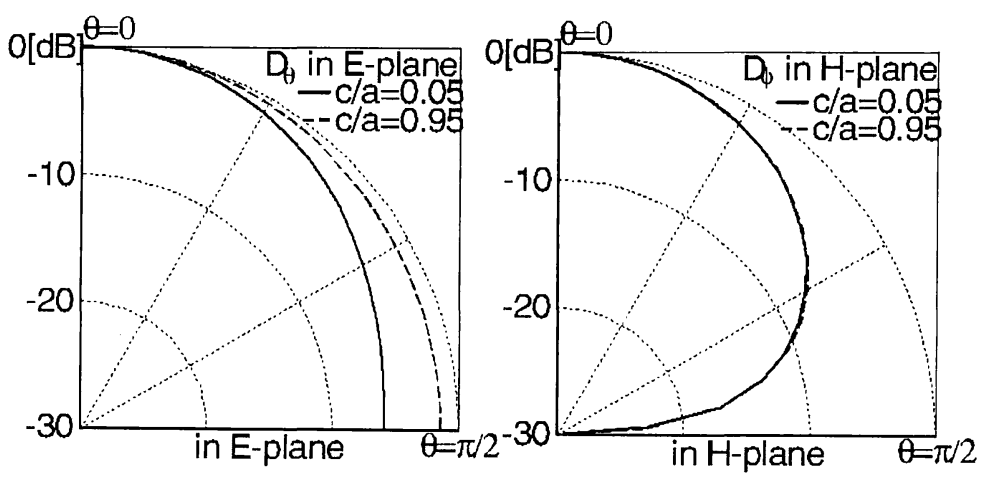

Fig. 3: Radiation pattcrns 